\title{
Modelos experimentais para - estudo do diabetes tipo 1
}

\section{Animal models for type 1 diabetes studies}

\author{
Vanessa R. Kirsten ${ }^{1}$, Patrícia Sesterheim², David Saitovitch ${ }^{3}$
}

\begin{abstract}
RESUMO
Os modelos animais de diabetes têm sido usados extensivamente na obtenção do esclarecimento sobre esta doença. $O$ objetivo deste estudo foi realizar uma revisão bibliográfica sobre os principais modelos experimentais para o estudo do diabetes mellitus. Dentre os modelos experimentais para o estudo do diabetes, existem os modelos induzidos quimicamente por aloxana e streptozotocina, sendo que a dose utilizada depende da espécie do animal e do seu peso. Além disso, existem dois excelentes modelos de diabetes espontâneo: os ratos BB (Biobreading) e os camundongos NOD (Non Obese Diabetic). Os camundongos NOD são o modelo mais estudado de doença espontânea auto-imune órgão-específico em todo o mundo. As razões para a preferência deste modelo incluem um genoma bem definido, maior quantidade de reagentes monoclonais para a análise de componentes do sistema imune e um custo razoavelmente baixo, comparado com a utilização de ratos. Estes camundongos exibem autoimunidade espontânea com destruição das ilhotas pancreáticas, de forma semelhante à observada em humanos. A destruição auto-imune é caracterizada por insulite e infiltrado leucocitário nas ilhotas pancreáticas. Esta infiltração é composta predominantemente por células dendríticas, macrófagos, por células TCD4, TCD8 e células B. Os fatores ambientais em conjunto com a genética, claramente modificam a incidência do diabetes tipo 1 nos modelos experimentais espontâneos. A suscetibilidade destes camundongos é poligênica e ambiental, enfatizando condições de habitação, sanitárias, dietéticas e de gênero. A incidência de diabetes em camundongos NOD é aproximadamente quatro vezes maior em fêmeas do que em machos. As informações obtidas através deste excelente modelo animal podem ser relevantes para $O$ entendimento do processo da doença nos humanos.
\end{abstract}

Palavras-chave: Diabetes Mellitus Experimental. Modelos de Animal Experimental. Camundongos Diabéticos não Obesos.

\footnotetext{
${ }^{1}$ Professora do Curso de Nutrição do Centro Universitário Franciscano (UNIFRA), Doutoranda em Saúde da Criança e do adolescente (UFRGS) Mestre em Ciências da Saúde pela Pontifícia Universidade Católica do Rio Grande do Sul (PUCRS), Especialista em Nutrição Clínica pela UNISINOS, Nutricionista graduada pelo Centro Universitário Franciscano (UNIFRA).

${ }^{2}$ Bióloga (PUCRS); Mestre em Clínica Médica/Nefrologia (UFRGS); Doutora em Ciências da Saúde/Nefrologia (PUCRS).

${ }^{3}$ Médico pela Universidade Federal do Rio Grande do Sul (UFRGS), Mestre em Medicina (Nefrologia) pela Universidade Federal de São Paulo (UNIFESP), Doutor em Imunologia de Transplantes University of Oxford. Professor adjunto da Pontifícia Universidade Católica do Rio Grande do Sul (PUCRS).
}

Correspondência: Prof - Vanessa Ramos Kirsten Centro Universitário Franciscano - Curso de Nutrição Rua Silva Jardim,1175. Bairro: Centro 97010-491 Santa Maria/RS E-mail: vanessark@unifra.br

Artigo recebido em 17/07/2008 Aprovado em 29/01/2010 


\section{Introdução}

O Diabetes Mellitus (DM) é uma doença metabólica, resultante de defeitos na secreção e/ou ação da insulina que levam à hiperglicemia. ${ }^{1} \mathrm{O}$ Diabetes Mellitus tipo 1 (DM1) é caracterizado por ser autoimune pelo estágio pré-clínico ter a presença de autoanticorpos contra constituintes da célula beta pancreática. ${ }^{2}$ Marcadores como o anticorpo anti-ilhota (ICAs), anti-insulina (IAAs), ácido glutâmico descarboxilase (GAD-65) e as tirosinas fosfatases IA-2 e 1A-2B, estão relacionados com o desenvolvimento do DM1. ${ }^{3} \mathrm{Ge}-$ ralmente, pelo menos um destes marcadores está presente em 85-90\% dos indivíduos com hiperglicemia de jejum, diagnosticado no início da doença. ${ }^{1}$

A história natural do DM1 inclui quatro estágios distintos: (I) pré-clínico: auto-imunidade dirigida às células beta, com uma diminuição aguda e progressiva da resposta insulínica à glicose intravenosa ou oral; (II) início do diabetes clínico; (III) remissão transitória; (IV) diabetes associado com complicações agudas, crônicas (retinopatia, nefropatia e vasculopatia) e morte. ${ }^{4,5}$

O tratamento intensivo com insulina, efetivamente, adia o início da progressão de doenças crônicas secundárias em pacientes com DM1. ${ }^{6}$ Novas alternativas de aplicação de insulina, como o uso de bombas de insulina de ação rápida, por infusão contínua subcutânea ou insulinas de ação ultra prolongada vêm sendo desenvolvidas, e buscam oferecer maior liberdade e qualidade de vida ao paciente diabético. ${ }^{7}$

Os modelos animais de diabetes têm sido usados extensivamente na obtenção do esclarecimento sobre esta doença. ${ }^{8}$ Eles têm fornecido inúmeros benefícios para a saúde, incluindo o tratamento de complicações oculares ou vasculares e o desenvolvimento das técnicas de transplante de pâncreas, evidenciando o aumento do entendimento dos processos imunológicos envolvidos na etiologia do diabetes tipo $1 .{ }^{9}$

Diante da necessidade maiores estudos experimentais sobre o diabetes, o objetivo deste estudo foi realizar uma revisão bibliográfica sobre os principais modelos experimentais para o estudo do diabetes mellitus, apontando o melhor modelo para determinados tipos de pesquisa.

\section{Metodologia}

O artigo foi elaborado através de uma revisão bibliográfica em artigos indexados em bases de dados como Scielo e Pubmed, com as seguintes palavraschaves: "diabetes" e "modelos experimentais" e "camundongos NOD".

\section{Modelos experimentais em diabetes}

Existem modelos experimentais para a pesquisa do DM1 que são utilizados largamente e que podem ser classificados em 2 tipos: diabetes mellitus induzido quimicamente e diabetes mellitus espontâneo.

\section{Diabetes experimental induzido quimicamente}

A indução química do diabetes, em animais experimentais, ocorre após a destruição química seletiva das células beta pancreáticas. As substâncias mais usadas para esta indução em ratos, camundongos e coelhos são a Alloxana e a Estreptozotocina (STZ). A dose destas drogas para indução do diabetes depende da espécie do animal e do seu peso. ${ }^{10}$

A STZ, na dosagem de 160 a $250 \mathrm{mg} / \mathrm{kg}$, apresenta efeito citotóxico direto nas células beta de camundongos. A STZ causa dano no ácido desoxirribonucléico (DNA), depletando a Nicotina Adenina Dinucleotídeo $\left(\mathrm{NAD}^{+}\right)$, que inibe a biossíntese e a secreção de insulina e, deste modo iniciam a morte das células beta, através da depleção de energia. Em doses sub-diabetogênicas $(40 \mathrm{mg} / \mathrm{kg}$ ) a STZ pode produzir insulite pancreática, com morte progressiva das células beta levando também ao diabetes mellitus. $\mathrm{O}$ aparecimento de lesão inflamatória nas ilhotas sugere que, múltiplas baixas (por exemplo $40 \mathrm{mg} / \mathrm{kg}$ ) doses de STZ agem pela iniciação de reação imune mediada por células. ${ }^{11}$

Muitas investigações foram realizadas para verificar o real efeito diabetogênico desta droga nas células beta, e verificaram que a lesão destas células in vitro resulta na descarga transitória de glutamato descarboxilase (enzima da via de síntese de ácido gamaaminobutírico - GABA) no meio extracelular, e foi proposto que esta enzima serviria como marcador para os processos tóxicos e como um auto-antígeno potencial para a reatividade imune. ${ }^{12}$

De modo geral, a sensibilidade em cães é máxima (50\% de mortalidade - dose de $50 \mathrm{mg} / \mathrm{kg}$ ) e mínima em camundongos (dose diabetogênica efetiva em média de $200 \mathrm{mg} / \mathrm{kg})^{12}$.

AAlloxana é mais frequentemente administrada por aplicações intravenosas, intraperitoneais ou subcutâneas. As ilhotas humanas são mais resistentes 
a alloxana do que as de ratos e camundongos. A dose intravenosa mais frequentemente usada para induzir diabetes em ratos é $65 \mathrm{mg} / \mathrm{kg}$. A dose intraperitoneal ou subcutânea efetiva deve ser 2 a 3 vezes maior do que este valor, pois abaixo de $150 \mathrm{mg} / \mathrm{kg}$ pode ser insuficiente para causar diabetes em ratos que, em jejum, se tornam mais suscetíveis à ação da droga. ${ }^{10}$

No estudo de Lerco e colaboradores, ${ }^{13}$ a dose de $42 \mathrm{mg} / \mathrm{kg}$ de peso corporal por via endovenosa em ratos Wistar, foi capaz de desenvolver alterações clínicas e laboratoriais bem definidas em $78 \%$ dos animais, como elevação da ingestão hídrica e da diurese, glicemia acima de $300 \mathrm{mg} / \mathrm{dL}$ e glicose urinária maior que $300 \mathrm{mg} / \mathrm{dL}$.

\section{Diabetes experimental espontâneo}

Os animais que desenvolvem espontaneamente o diabetes insulino-dependente têm sido estudados em amplo espectro por 2 fatores patogenéticos que se complementam: os defeitos imunológicos e a predisposição genética. Existem várias espécies, como o Hamster Chino, o coelho Branco da Nova Zelândia e o cachorro Keshond, porém estes animais não são suficientemente caracterizados e convenientes para serem comparáveis com o DM1 dos seres humanos. ${ }^{14}$

Entretanto, o DM1 possui dois excelentes modelos animais espontâneos para a doença: os ratos BB (Biobreading) e os camundongos NOD (Non Obese Diabetic). ${ }^{8,15,16,17}$

Nos ratos $\mathrm{BB}$, descobertos no Laboratório BioBreeding de Ottawa (Canadá), os sintomas aparecem ao redor dos 3 meses de idade. A incidência cumulativa de diabetes nestes ratos pode ser de $100 \%$ se mantidos em ambiente livre de patógenos. ${ }^{18}$ As manifestações incluem hipoinsulinemia, hiperglicemia (20 a 30mmol/L), glicosúria, perda de peso, polidipsia, poliúria e cetoacidose. Tem-se reportado tratamentos aplicados a estes ratos que interferem na resposta imunológica e previnem o diabetes, como timectomia neonatal, irradiação linfóide total, tratamento com soro antilinfocítico ou ciclosporina. ${ }^{14}$

A predisposição genética destes animais é demonstrada através de estudos que empregam o cruzamento entre linhagens distintas entre si, de ratos BB com outras linhagens co-sanguíneas, e identificaram 3 genes: [I] iddm1 - Lyp (linfopenia) autossômica recessiva, localizado no cromossomo 4 e induz a ausência de células $\mathrm{T}$ periféricas das subpopulações $\mathrm{CD}^{+} \mathrm{e}$ $\mathrm{RT}^{+}$desde o nascimento, [II] iddm2 - MHC (Complexo Principal de Histocompatibilidade) presente no cromossomo 20, que induz suscetibilidade a insulite e ao diabetes, [III] iddm 3 confere resistência ao diabetes. Fenotipicamente apresentam linfopenia e um fenômeno poliendócrino como a tireoidite auto-imune. ${ }^{14}$

A imunopatogênese do diabetes nos ratos BB tem demonstrado a importância das células $\mathrm{T}$ no processo da doença, assim como, os diferentes caminhos na manipulação do sistema imune para a prevenção do diabetes. ${ }^{18}$

\section{Camundongos NOD}

Os camundongos NOD são o modelo mais estudado de doença espontânea auto-imune órgão-específico em todo o mundo. ${ }^{19,20,21}$ As razões para a preferência deste modelo incluem um genoma bem definido, maior quantidade de reagentes monoclonais para a análise de componentes do sistema imune e um custo razoavelmente baixo, comparado com a utilização de ratos. ${ }^{17}$

\section{Background (linha evolutiva genética) da li- nhagem NOD}

Os camundongos NOD foram descobertos em 1974, no Laboratório de Investigações Shinogui, em Osaka - Japão. ${ }^{14}$ Possuem fenótipo albino (figura 1) e são procedentes de uma derivação da subllinhagem Jc 1 - ICR outbread que desenvolvia catarata. ${ }^{17}$

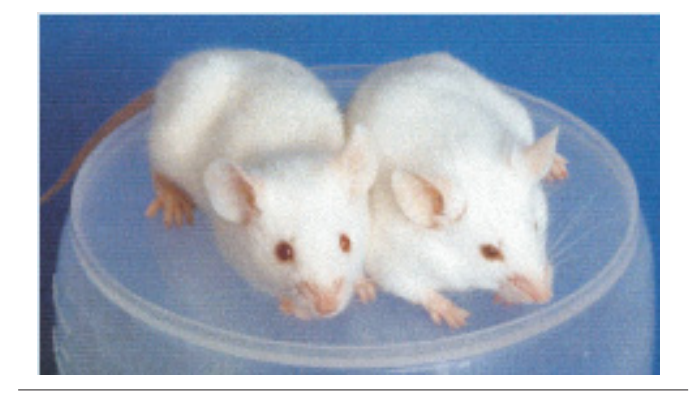

Figura 1. Camundongos NOD17.

Segundo o Laboratório Jackson, na $6^{\mathrm{a}}$ geração, os progenitores dos futuros NOD/Shi foram acasalados inbread com camundongos livres de catarata, mas com níveis elevados de glicemia de jejum. $\mathrm{Na} 13^{\circ}$ geração, os progenitores foram separados da nova linhagem NOD/Shi. Os níveis plasmáticos elevados de glicose continuaram a ser a base para a seleção da última linhagem, enquanto que seus progenitores - da geração 13 - foram selecionados na base de níveis normais de glicose sanguínea plasmática. Em 1974, na $20^{\circ}$ geração, uma fêmea de linhagem normo- 
glicêmica desenvolveu espontaneamente diabetes mellitus, depeleção de insulina sérica e insulite. A reprodução seletiva da linhagem desta fêmea diabética produziu a linhagem "Nonobese Diabetic" - NOD. ${ }^{22}$

\section{Processo inflamatório}

Estes camundongos exibem autoimunidade espontânea que causa diabetes através da destruição das células produtoras de insulina, de forma semelhante à observada em humanos. ${ }^{23}$ A destruição auto-imune é caracterizada por insulite, infiltrado leucocitário nas ilhotas pancreáticas. Esta infiltração é composta predominantemente por células dendríticas, macrófagos, por células $\mathrm{T}\left(\mathrm{CD}^{+}{ }^{+}\right.$e $\left.\mathrm{CD} 8^{+}\right)$e células $\mathrm{B}$ - e ocorre do ducto perivascular para regiões periféricas das ilhotas pancreáticas de Langerhans (peri-insulitis). ${ }^{19}$ Algumas células NK (Natural Killer) também são achadas no infiltrado. $^{24}$

Este estágio é acompanhado por uma lenta, progressiva e seletiva destruição das células beta, mediada principalmente, por células T, iniciam-se em 3-4 semanas e estendendo-se por 4-6 meses de idade. ${ }^{19}$ Segundo Gross e colaboradores, ${ }^{25} \mathrm{O}$ infiltrado nas ilhotas é similar ao que é observado nos pacientes com início recente do diabetes tipo 1 . No entanto, para Atkinson, ${ }^{17}$ a insulite em pacientes diabéticos de início agudo é muito diferente da encontrada nas ilhotas dos NOD.

Este infiltrado progride e invade as ilhotas (insulite) em poucas semanas, levando a um quadro severo de insulite em 10 semanas de idade. ${ }^{26}$

Paralelamente, há liberação de citocinas próinflamatórias como Fator de Necrose Tumoral $\alpha$ (TNF- $\alpha$ ), Interferon-Gama (IFN- $\gamma$ ) e Interleucina 1 beta (IL-1 $\beta$ ). Essas citocinas, secretadas pelas células apresentadoras de antígeno (APC) e células T, podem favorecer a iniciação e progressão da resposta auto-imune. Além disso, podem ser finalmente reguladas por outras citocinas e quimiocinas numa complexa rede de interações recíprocas. ${ }^{26}$

Uma das peculiaridades da linhagem é a acumulação de algumas células $\mathrm{T}$ auto-reativas nos órgãos linfóides periféricos, pâncreas e glândulas salivares submandibulares. Este acúmulo de linfócitos $\mathrm{T}$ possibilita o reflexo de baixos níveis de IL-2 e a resistência de timócitos e células T periféricas para a indução de apoptose. ${ }^{17}$ Os camundongos NOD também desenvolvem doença auto-imune generalizada, afetando múltiplos órgãos e tecidos, incluindo salivar, lacrimal, tireóide, paratireóide, adrenal, testículos e hemáceas. ${ }^{16,17}$
Somente após a destruição de pelo menos $95 \%$ das células beta produtoras de insulina é que o diabetes se manifesta nos camundongos NOD. ${ }^{14}$

Similar ao processo que acontece nos humanos, os camundongos NOD geralmente expressam autoanticorpos anti-insulina no soro antes de apresentarem hiperglicemia. ${ }^{21}$ De acordo com Gross e colaboradores, ${ }^{25}$ a variedade de autoanticorpos contra proteínas das ilhotas pancreáticas, incluindo insulina, ácido glutâmico descarboxilase (GAD) e ICA512, foi identificada tanto no modelo animal quanto no homem.

A insulite por sua vez, pode ser prevenida mediante vários procedimentos imunomoduladores, que compreendem a timectomia neonatal, transplante de medula óssea, irradiação, transfusão de sangue, ciclosporina A, corticosteróides, nicotinamida, eliminação de radicais livres ${ }^{14}$ e tratamento com anticorpo monoclonal anti-CD4 e anti-CD8. ${ }^{19}$

\section{Características do diabetes no modelo ex- perimental NOD}

Embora todos os camundongos NOD desenvolvam insulite, isto não é sempre acompanhado de diabetes..$^{21}$ Neste modelo experimental, o início do diabetes é marcado por glicosúria moderada e glicemia maior que $250 \mathrm{mg} / \mathrm{dl}$. Eles são hipoinsulinêmicos e hiperglucagonêmicos, confirmando a destruição seletiva das células beta pancreáticas. ${ }^{25}$ A glicosúria e a hiperglicemia tornam-se progressivamente mais severas por volta da $34^{\mathrm{a}}$ semana, quando a perda de peso, a polidipsia e a poliúria ocorrem. Sem o tratamento com insulina exógena, os camundongos diabéticos tornam-se severamente hiperglicêmicos e cetonêmicos, mas eles não se tornam cetoacidóticos. ${ }^{27}$

Na maior parte de colônias criadas em ambiente SPF (livre de patógenos), os camundongos NOD sem tratamento sobrevivem de 3 a 4 semanas após a primeira detecção de glicosúria. ${ }^{19}$

\section{Suscetibilidade para o desenvolvimento de diabetes nos camundongos NOD}

Os fatores ambientais em conjunto com a genética, claramente modificam a incidência do diabetes tipo 1 nos modelos experimentais espontâneos. A suscetibilidade destes camundongos é poligênica e ambiental, enfatizando condições de habitação (ambiente gnobiótico), sanitárias, dietéticas e de gênero. ${ }^{20,22}$

Isto se deve ao fato que a diabetogênese nos camundongos NOD é uma consequência hereditária de imudeficiência dentro do complexo poligênico. A 
penetrância desta suscetibilidade é fortemente influenciada pelo ambiente físico, particularmente a dieta e a exposição a patógenos microbianos. Uma das características mais fascinantes dos camundongos NOD é a estimulação do sistema imune pelos patógenos ambientais que levam ao desenvolvimento de um sistema imune mais normal e menos suscetível ao diabetes. Assim, estes animais precisam ser alocados em condições livre de patógenos (SPF) para expressar o fenótipo do diabetes. ${ }^{28}$

\section{a) Genética}

A herança genética da suscetibilidade para a doença em humanos (DM1) e nos camundongos NOD é poligênica. ${ }^{29}$ Os alelos de classe II do MHC constituem um dos mais importantes fatores de risco genéticos para a suscetibilidade ao DM1. Os camundongos NOD são homozigotos para o haplótipo $\mathrm{H}-2^{\mathrm{g} 7}\left(\mathrm{~K}^{\mathrm{d}}\right.$, $\mathrm{I}-\mathrm{A}^{\mathrm{g} 7}, \mathrm{I}-\mathrm{E}^{\text {null }}, \mathrm{D}^{\mathrm{b}}$ ) que mapeia o locus idd-1 no cromossomo 17, e contribui para disfunções severas de células apresentadoras de antígenos (APC), que podem promover o desenvolvimento de células T- autoreativas às ilhotas. ${ }^{19,30}$ Esta região é necessária, mas não suficiente, para o desenvolvimento visível do diabetes. ${ }^{29}$

Recentemente, foram correlacionadas as sequências codificadoras da Interleucina 2 (IL-2) e o polimorfismo da molécula CTLA-4 no desenvolvimento de diabetes em camundongos NOD. ${ }^{30}$

\section{b) Dietética}

Fatores dietéticos modificam o desenvolvimento do diabetes autoimune em modelos animais de diabetes (camundongos NOD e os ratos BB). ${ }^{31}$

Em alguns estudos, proteínas ou peptídeos constituem fatores desencadeantes para o diabetes, além de alguns componentes das rações padronizadas para roedores, que contém trigo, soja e alfafa, também podem desencadear diabetes tanto nos camundongos NOD como nos ratos BB. A ingestão de leite ou albumina sérica bovina (BSA) durante a infância tem sido sugerida como importante fator desencadeante de diabetes tipo 1 em humanos. No entanto, estudos em animais são divergentes a este respeito. ${ }^{14,18}$

Além desses componentes, foi verificado que, nestes camundongos, a introdução precoce de dieta isenta de glúten, influencia o início e a incidência de diabetes, assim como a insulite e o número de linfócitos na mucosa intestinal, retardando significativamente o diabetes. ${ }^{32}$ Dietas restritas em ácidos graxos essen- ciais, como o ácido araquidônico, também previnem o desenvolvimento do diabetes nestes animais. ${ }^{14}$

De acordo com Mathieu e colaboradores, ${ }^{33} \mathrm{ca}-$ mundongos NOD tratados precocemente (começando aos 3 dias de idade até os 70 dias de vida), com vitamina $\mathrm{D}$ ou com 1,25-dihidroxivitamina $\mathrm{D}_{3}\left(1,25(\mathrm{OH})_{2} \mathrm{D}_{3}\right)$ desenvolvem diabetes em menor quantidade. $\mathrm{O}$ efeito da deficiência de vitamina D é maior que o da suplementação, sugerindo um papel importante para esta molécula na patogênese desta patologia.

\section{c) Exposição a microorganismos}

Teoricamente, infecções por vírus podem contribuir para o aumento ou diminuição da incidência do diabetes tipo 1. ${ }^{18,34}$ Em modelos experimentais, o diabetes pode ser prevenido nos camundongos NOD pela infecção em idade jovem com micobactéria, vírus da coriomeningite linfocítica, vírus da hepatite murina, vírus da encefalomiocardites. ${ }^{35}$ De acordo com Christen e colaboradores, ${ }^{36}$ injeções com Coxsackie vírus não somente melhoram, mas também previnem a doença nos camundongos NOD.

Segundo Leiter, ${ }^{27}$ a imunoestimulação geral deve ser subjacente à proteção ao desenvolvimento de diabetes, porque o tratamento de NODs pré-diabéticos com vários tipos de imunomoduladores exógenos, incluindo citocinas (IL-1, TNF, IL-2, IL-4), previne em geral o aparecimento desta doença.

Camundongos NOD vacinados com o Bacillus Calmette Guérin (BCG) durante as primeiras semanas de vida previnem o aparecimento do diabetes. $\mathrm{Da}$ mesma forma, as enterotoxinas estafilocócicas também previnem o aparecimento do diabetes nestes animais. ${ }^{14}$

\section{d) Estresse Crônico}

$\mathrm{O}$ estresse crônico nos camundongos NOD (como rotação, vibração, limitação de espaço) entre a sexta e a oitava semana de vida, assim como repetidas injeções contendo salina diminuem a incidência de diabetes em ambos os sexos. O estresse pré-natal acelera o início do diabetes. Suspeita-se que o estresse modula o desenvolvimento do diabetes por interações neuroendócrinas-imunes envolvendo glucocorticóides e citocinas. ${ }^{18}$

\section{e) Ambiental}

Para um melhor manejo em biotérios, os camundongos NOD, deverão estar em ambiente SPF, alocados em gaiolas com filtro e autoclavadas, em lo- 
cais com irradiação e fluxo laminar. ${ }^{28}$ Somente animais com boa qualidade microbiológica podem oferecer garantia de um resultado experimental sem interferências de outras variáveis, por isso as utilizações desses animais (em SPF) vêm crescendo à medida que os pesquisadores necessitam de resultados mais fidedignos de seus experimentos. ${ }^{37}$

Em camundongos NOD alocados em instalações padrão, o diabetes afeta primeiramente as fêmeas, com incidência entre $60-90 \%$ ao redor da 30 a semana de idade, em oposição aos machos os quais se tornam diabéticos em apenas $30 \%$ dos casos. A incidência cumulativa, entretanto, é reportada próximo de $100 \%$, independente do gênero. ${ }^{18}$

A transferência de machos NOD de biotério convencional, no Japão, para biotério SPF aumentou a incidência de diabetes nos machos de 6 para $70 \% .^{27}$

A incidência de diabetes nos camundongos NOD é muito divergente entre os laboratórios, conforme pode ser observado na Tabela 1.

No Biotério do Laboratório Jackson, a incidência relatada de diabetes é de $85 \%$ na colônia de fêmeas e $37 \%$ nos machos em 30 semanas de idade. ${ }^{22}$ Delovitch e Singh ${ }^{19}$ reportam que a incidência nas fêmeas varia de $80-90 \%$ enquanto nos machos de $10-40 \%$. De acordo com Hanafusa e colaboradores ${ }^{24}$ em 30 semanas de idade, a incidência de diabetes alcança $80 \%$ nas fêmeas e $20 \%$ nos machos.

A incidência de diabetes em NOD machos serve como um indicador útil da presença de fatores ambientais afetando a penetrância da linhagem na suscetibilidade para o diabetes. Expor camundongos NOD a uma variedade de vírus de murinos (por exemplo, vírus da encefalomiocarditis, hepatite, coriomeningite) previne o desenvolvimento de diabetes. ${ }^{27}$

Estes agentes infecciosos, aparentemente, protegem pelo fornecimento de imunoestimulação geral, devido ao tratamento de NOD pré-diabéticos com vários tipos de imunomoduladores exógenos, incluindo ACF e citocinas (IL-1, TNF- $\alpha$, IL-2, IL-4), todos evitam o desenvolvimento do diabetes. ${ }^{27}$

\section{f) Divergência entre os sexos}

A incidência de diabetes em camundongos NOD é aproximadamente quatro vezes maior em fêmeas do que em machos. ${ }^{18}$

Em análises de colônias NOD, em todo o mundo, verificou-se que a incidência cumulativa de diabetes na $30^{\circ}$ semana é mais variável: menor nos machos do que nas fêmeas. Embora algumas diferenças das colônias possam ser explicadas por divergências genéticas entre linhagens NOD separadas das colônias originais, estas parecem depender muito mais de fatores ambientais. ${ }^{29}$

Conforme Delovitch e Singh, ${ }^{19}$ as fêmeas NOD desenvolvem uma forma mais agressiva de insulite e a consequente incidência mais alta de diabetes comparadas aos machos. O início da doença pode ser acelerado em machos castrados. O inverso é observado em fêmeas tratadas com andrógenos. ${ }^{14,18}$ Estes achados sugerem um papel imunomodulador para os hormônios sexuais. Estas observações do modelo animal contrastam com as do diabetes tipo 1 em seres humanos, no qual não há diferença de incidência entre homens e mulheres. ${ }^{38}$

O fato dos camundongos NOD serem isogênicos, a necessidade de condições de habitação controlada, a facilidade para mudar a fisiologia natural através de manipulação genética e a relativa facilidade para a prevenção da doença tem causado alguns questionamentos sobre a qualidade do modelo. Fica claro que o curso do diabetes tipo 1 em humanos não será facilmente alterado, ao contrário do observado nos modelos murinos isogênicos, onde o risco genético é o mesmo para todos e, intervenções podem ser iniciadas em muitos estágios da doença. ${ }^{17}$

Assim, não se pode assumir que os modelos disponíveis de diabetes espontâneo em ratos e camundongos representem um equivalente à doença em humanos. ${ }^{17}$ Entretanto, estes parecem ser instrumentos importantes de pesquisa, principalmente, da patogênese e terapia do diabetes humano.

\section{Tabela 1}

Colônias de NOD e suas taxas de diabetes em ambiente SPF $^{18}$

\begin{tabular}{lcc}
\hline Colônias & Incidência de diabete & Referências \\
\hline NOD/Lt (Leiter) & $90 \%$ fêmeas, $83 \%$ machos & $(29)$ \\
NOD/Shi (Shionogi) & $70-80$ fêmeas, $<20 \%$ machos & $(30)$ \\
NOD/Wehi (Walter and Eliza Hall Institute) & $<10 \%$ Fêmeas, $<1 \%$ nos machos & $(31)$ \\
\hline
\end{tabular}




\begin{abstract}
The animal models of diabetes have been used extensively in obtaining the information on this disease. The objective of this study was a literature review on the main experimental models for the study of diabetes mellitus. Among the experimental models for the study of diabetes, the models are chemically induced by aloxan and streptozotocin, and the dose used depends on the species of the animal and its weight. Also, there are two excellent models of spontaneous diabetes: the BB rats (Biobreading) and NOD mice (Non Obese Diabetic). The NOD mice are the most studied model of spontaneous selfimmune disease-specific body in the world. The reasons for the preference genome of this model include a well-defined, greater quantity of monoclonal reagents for the analysis of components of the immune system and a reasonably low cost, compared with the use of rats. These mice exhibit spontaneous autoimmunity with destruction of pancreatic islets, in a manner similar to that seen in humans. The auto-immune destruction is characterized by insulite in pancreatic islets. This infiltration is composed predominantly of dendritic cells, macrophages, CD4 T cells, CD8 cells and B. The environmental factors together with the genetics, clearly alter the incidence of type 1 diabetes in experimental models spontaneous. The susceptibility of these mice is genetics and environment, emphasizing adequate housing, health, diet and gender. The incidence of diabetes in NOD mice is about four times higher in females than in males. Information obtained through this excellent animal model may be relevant to the understanding of the process of the disease in humans.
\end{abstract}

Keywords: Diabetes Mellitus, Experimental. Experimental Animal Models. Non-Obese Diabetic Mice.

\section{Referências Bibliográficas}

1. The Expert Committe on the diagnosis and classification of diabetes mellitus. Report of the expert committee on the diagnosis and classification of diabetes mellitus. Diabetes Care. 2003; 26(Suppl.1):s5-20.

2. Cesarini PR, Mendonça $E$, Fernandes $V$ et al. Prevalência dos marcadores imunológicos anti-GAD e anti-IA2 em parentes de primeiro grau de diabéticos do tipo $1 \mathrm{em}$ amostra da população da grande São Paulo. Rev. Assoc. Med. Bras. (1992). 2003; 49(4): 395-400.

3. Koczawara K, Schenker M, Schmid S, Kredel K et al. Characterization of antibody responses to endogenous and exogenous antigen in the nonobese diabetic mouse. Clin. Immunol. 2003; 106: 155-62.

4. Rewers M, Klingensmith GJ. Prevention of type 1 Diabetes. Diabetes Spectr. 1997; 10(4): 282-92.

5. Joshi N, Caputo GM, Weitekamp MR, Karchmer AW. Infectious in patients with diabetes mellitus. N. Engl. J. Med. 1999; 342(25): 1906-12.

6. Gotoh M, Sato Y, Abe T, Kanazawa Y. New approaches for successfull islet transplantation. J. Hepatobiliary. Pancreat. Surg. 2000; 7: 358-63.

7. Dills DC. Novos aspectos da terapêutica com insulina no diabetes tipo 1 e tipo 2. Current Diabetes Reports Latin America 2002; 2:125-32.

8. Buschard K. Meet a research Group Diabetes Research Group, Bartholin Institutted, Kommunehospitalet, Conpenhagen, Denmark. Scand J Lab. Anim Sci 2001; 28(1).

9. Sieher FE, Traystman RJ. Ethical Issues Involved in the Development of Animal Models for Type I Diabetes: Models of Type I Diabetes - Part One. Institute for Laboratory Animal Research Journal 1993; 35(1): 1-6.

10. Szkudelski T. The mechanism of alloxan and streptozotocin action in B cells of the Rat pancreas. Physiol Res. 2001, 50:536-46.
11. Rayat GR, Singh B, Korbutt GS, Rajotte RV. Single injection of insulin delays the recurrence of diabetes in syngeneic islettransplanted diabetic NOD mice. Transplantation 2000. 70(6):976-85.

12. Furlan MMDP. A estreptozotocina como agente diabetogênico. Arq Ciênc Saúde Unipar 2001; 5(2): 197-201.

13. Lerco MM, Spadella CT, Machado JLM, Schellini SA, Padovani CR. Caracterização de um modelo experimental de Diabetes Mellitus induzido pela aloxana em ratos. Estudo Clínico e laboratorial. Acta cir bras. 2003; 18 (2): 132-42.

14. Hernandorena BH, Gonzales JCR, Garcia JCR. Animales de Laboratório en la Endocrinología: Biomodelos de la Diabetes Mellitus tipo 1. Rev cuba endocrinol. 2001; 12(3): 168-77.

15. Leiter EH, Herrath Mv. Animal models have little to teach us about Type 1 diabetes: 2. In opposition to this proposal. Diabetologia. 2004; 47: 1657-60.

16. Delarche FH. Is pancreas development abnormal in the nonobese diabetic mouse, a spontaneous model of type I diabetes? Braz j med biol res. 2001; 34: 437-47.

17. Atkinson MA, Leiter EH. The NOD mouse model of type 1 diabetes: As good as it gets? Nat. med. 1999 Jun; 5(6): 601-4.

18. Bieg S, Lernmark A. Animal Models for Insulin-dependent diabetes mellitus. In: Totowa NJ. Contemporary endocrinology autoimmune endocrinopathies. Humana Press 1999: 113-39.

(19. Delovitch TL, Singh B. The Nonobese Diabetic Mouse as a Model of Autoimmune Diabetes: Immune Dysregulation Gets the NOD. Immunity. 1997; 7: 727-38.

20. Rosmalen JGM, Leenen PJM, Pelegri C, Drexhage HA, HomoDelarche $\mathrm{F}$. Islet abnormalities in the pathogenesis of autoimmune diabetes. Trends endocrinol. metab. 2002;13(5): 209-14.

21. Eisenbarth GS. Animal Models of type 1 Diabetes: Genetics and immunological function. IN:L Type I diabetes: Molecular, Cellular, and Clinical Immunology [capítulo de livro on line] [capturado 2005 Ago 02]; Chapter 3: Disponível em: http:// www.uchsc.edu/misc/diabetes/oxch3.html 
22. Leiter EH, Staff S. NOD/Ltj Mice Available. [página on line]1993 [capturado 2004 maio 04]; Disponível em: http:://www.jax.org.

23. Kodama S, Kuhtreiber W, Fujimura S, Dale EA, Faustman DL. Islet regeneration during the reversal of autoimmune diabetes in NOD mice. Science 2003; 302: 1223-7.

24. Hanafusa T, Miyagawa J, Nakajima H, Tomita K, Kuwajima M et al. The NOD mouse. Diabetes Res. Clin. Pract. 1994; 24S: S307-11.

25. Gross DJ, Weiss L, Reibstein I, Hedlund G, et al. The immunomodulator Linomide: role in treatment and prevention of autoimmune diabetes mellitus. Int. Immunopharmacol. 2001; 1: 1131-9.

26. Anderson MS, Bluestone JA. The NOD mouse: A Model of Immune Dysregulation. An. Rev. Immunol. 2005; 23: 447-85.

27. Leiter $\mathrm{EH}$. The NOD Mouse: a model for analyzing the interplay between heredity and Environment in development of autoimmune disease. Institute for Laboratory Animal Research Journal Models of type I diabetes - part one. 1993; 35(1): 4-14.

28. Leiter EH. The NOD mouse: A model for Insulin-Dependent Diabetes Mellitus. Current Protocolls in Immunology. [PDF on line] 1997 [capturado em 2004 nov 10]; 15.9.1. Suplement 24. Disponível em www.jax.org/t1dr/NOD_CPI_mod.pdf.

29. Bowman MA, Leiter EH, Atkinson, MA. Prevention of Diabetes in the NOD mouse: Implications for Therapeutics Intervention in Human Disease.Immunol. today. 1994; 15(3): 115-20.

30. Aoki CA, Borchers AT, Ridgway WM, Keen CL, Ansan AA, Gershwin ME. NOD mice and autoimmunity. Autoimmun. Rev. 2005; 4: 373-9.
31. Vaarala O. Intestinal Immunity and Type 1 Diabetes. J. Pediatr. Gastroenterol. Nutr. 2004; 393):S732-3.

32. Funda DP, Kaas A, Bock T, Tlaskalova-Hogenova $\mathrm{H}$, Buschard K. Gluten-free diet prevents diabetes in NOD mice. Diabetes metab. res. 1999; 15:323-327.

33. Mathieu C, Etten EV, Decallonne B, Guilietti A, Gysemans C, Bouillon R, Overbergh L. Vitamin D and 1,25-dihydroxyvitamin D3 as modulators in the immune system. J. steroid biochem. mol. biol. 2004; 89-90: 449-52.

34. Salminen KK, Vuorinen T, Oikarinen S, Helminen M, Simell S et al. Isolation of enterovirus strains from children with preclinical Type 1 diabetes. Endocrinology and Metabolism. 2004; 21:156-64.

35. Saravia -Fernadez F, Durant S, el Hasnaoui A, Dardenne M, Homo-Delarche F. Environmental and experimental procedures leading to variations in the incidence of diabetes in the nonobese diabetic (NOD) mouse. Autoimmunity. 1996; 24: 113-21.

36. Christen U, Juedes A, Homann D, Herrath MGV. Viraly induced inflammation and therapeutic avenues in type 1 diabetes. Endocrinol. Metab. Clin. North Am. 2004; 33: 45-58.

37. Gui Mi Ko, Andersen ML, D’Almeida V, Kawakami R, Martins PJF, Magalhães JE, et al. Princípios Éticos e Práticos do Uso de Animais de Experimentação. São Paulo: UNIFESP; 2004.

38. Whitacre CC. Sex differences in autoimmune disease. Nat. Immunol. 2001; 2: 777-780. 